\title{
Acquisition of Cross-Resistance to Bedaquiline and Clofazimine following Treatment for Tuberculosis in Pakistan
}

\author{
(D) Arash Ghodousi, ${ }^{a}$ Alamdar Hussain Rizvi, ${ }^{b}$ Aurangzaib Quadir Baloch, ${ }^{c}$ Abdul Ghafoor, ${ }^{c}$ Faisal Masood Khanzada, \\ Mehmood Qadir,'b (D) Emanuele Borroni, ${ }^{a}$ (D) Alberto Trovato, ${ }^{a}$ Sabira Tahseen, ${ }^{b}$ (D) Daniela Maria Cirillo ${ }^{a}$ \\ aEmerging Bacterial Pathogens Unit, Division of Immunology, Transplantation and Infectious Diseases, IRCCS San Raffaele Scientific Institute, Milan, Italy \\ bNational TB Reference laboratory, National TB Control Program, Islamabad, Pakistan \\ cNational TB Control Program, Islamabad, Pakistan
}

ABSTRACT We report on the first six cases of acquired resistance to bedaquiline in Pakistan. Seventy sequential isolates from 30 drug-resistant-tuberculosis patients on bedaquiline-containing regimens were retrospectively tested for bedaquiline resistance by MIC testing and by the detection of mutations in relevant genes. We documented cases failing therapy that developed specific mutations in Rv0678 and had increased MICs associated with cross-resistance to clofazimine during treatment. This study underlines the relevance of surveillance programs following the introduction of new drugs.

KEYWORDS bedaquiline, clofazimine, whole-genome sequencing, XDR, antibiotic resistance, multidrug resistance, tuberculosis
Citation Ghodousi A, Rizvi AH, Baloch AQ Ghafoor A, Khanzada FM, Qadir M, Borroni E, Trovato A, Tahseen S, Cirillo DM. 2019. Acquisition of cross-resistance to bedaquiline and clofazimine following treatment for tuberculosis in Pakistan. Antimicrob Agents Chemother 63:e00915-19. https://doi.org/10 .1128/AAC.00915-19.

Copyright $\odot 2019$ Ghodousi et al. This is an open-access article distributed under the terms of the Creative Commons Attribution 4.0 International license.

Address correspondence to Daniela Maria Cirillo, cirillo.daniela@hsr.it.

S.T. and D.M.C. contributed equally to this article.

Received 6 May 2019 Returned for modification 24 May 2019 Accepted 23 June 2019

Accepted manuscript posted online 1 July 2019

Published 23 August 2019

At SRL-Milan, all strains underwent MIC testing for bedaquiline in $7 \mathrm{H} 11$ medium, and 


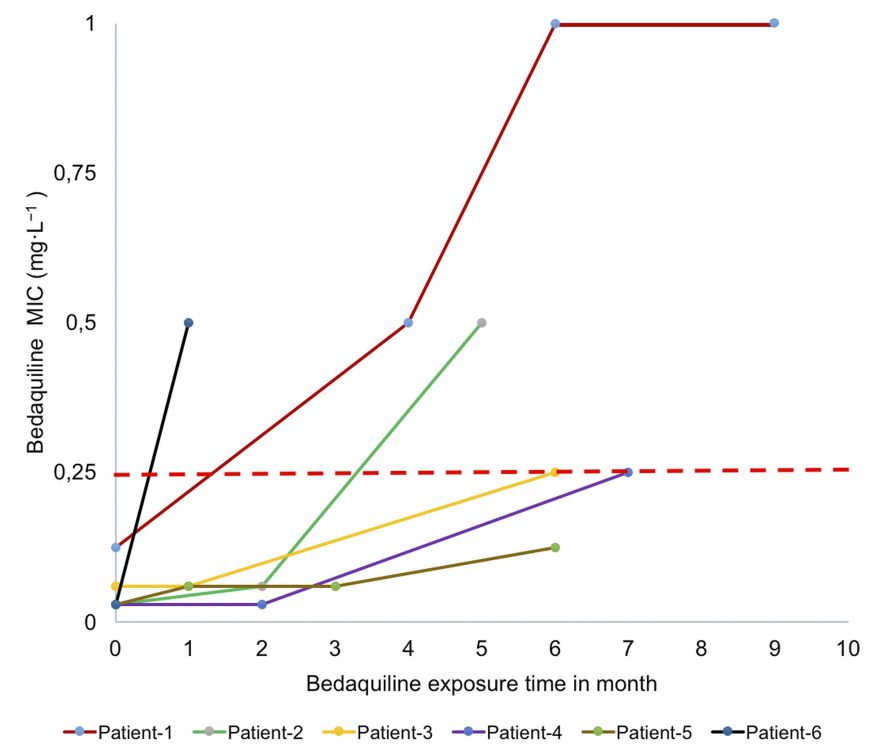

FIG 1 The MICs of bedaquiline performed in $7 \mathrm{H} 11$ medium in patients under treatment with bedaquiline-containing regimens at different time points. The dashed line in red shows the currently identified critical concentration for bedaquiline in $7 \mathrm{H} 11$ medium by the WHO (13).

for all isolates showing an increased MIC compared to the baseline isolate, further MIC testing for bedaquiline and clofazimine was conducted using the Bactec MGIT960 (BD, Franklin Lakes, NJ, USA) (6). The H37Rv (ATCC 27294) strain was used as a susceptible control in MIC testing. Bedaquiline dry powder was supplied by Janssen-Pharmaceutica (Beerse, Belgium). WGS was carried out with the Illumina Nextera-XT DNA sample preparation kit to prepare paired-end libraries of 150-bp read length to sequence on an Illumina NextSeq platform. Data analysis and single nucleotide polymorphism (SNP) calling were performed using the MTBseq-Pipeline on low-frequency detection mode (7). Genes associated with resistance to bedaquiline and/or clofazimine (atpE, Rv0678, pepQ, and Rv1979c) were screened for mutations (8-11).

We studied 70 isolates from patients ( $n=30 ; 8$ MDR, 15 pre-XDR, and 7 XDR) enrolled in bedaquiline-containing regimens. The sequential isolates tested included two strains from 22 patients, three from 6 patients, and four from 2 patients. All baseline strains included in the study were sensitive to bedaquiline. Six patients developed an increase in bedaquiline MICs in $7 \mathrm{H} 11$ medium (range, 0.125 to $>0.5 \mathrm{mg} \cdot$ liter $^{-1}$ ) during therapy, and five of them became resistant to bedaquiline according to the current critical concentration proposed for the drug (Fig. 1). The phenotypic/genotypic characteristics of Mycobacterium tuberculosis isolates from these six HIV-negative, unrelated pulmonary TB patients are summarized in Table 1.

Patient 1, a newly diagnosed rifampin-resistant TB case, was enrolled in second-line treatment. The patient remained culture positive and was subsequently diagnosed as a pre-XDR TB case, and after 5 months of second-line treatment, bedaquiline, clofazimine, and linezolid were added to the regimen. The patient continued to be culture positive, and 6 months later, delamanid was also added to the regimen; ultimately, treatment failure was declared. The first increase in bedaquiline MIC was seen at the fourth month of treatment $\left(0.5 \mathrm{mg} \cdot\right.$ liter $^{-1}$ in $\left.7 \mathrm{H} 11\right)$ concomitantly with WGS analysis showing an insertion (Ins) at nucleotide position 141 to 142 of Rv0678 (genomic position 779130). At month 9, the isolate became resistant to delamanid (MIC $>1 \mathrm{mg} \cdot$ liter $^{-1}$ ) (data not shown). WGS analysis confirmed the presence of the Rv0678 mutation and also showed a mutation in fgd1 (G104S), probably responsible for delamanid resistance.

Patients 2 and 3 had MDR TB with a history of failed second-line treatment taken for 20 and 17 months, respectively, both were then re-enrolled on a bedaquiline- and 


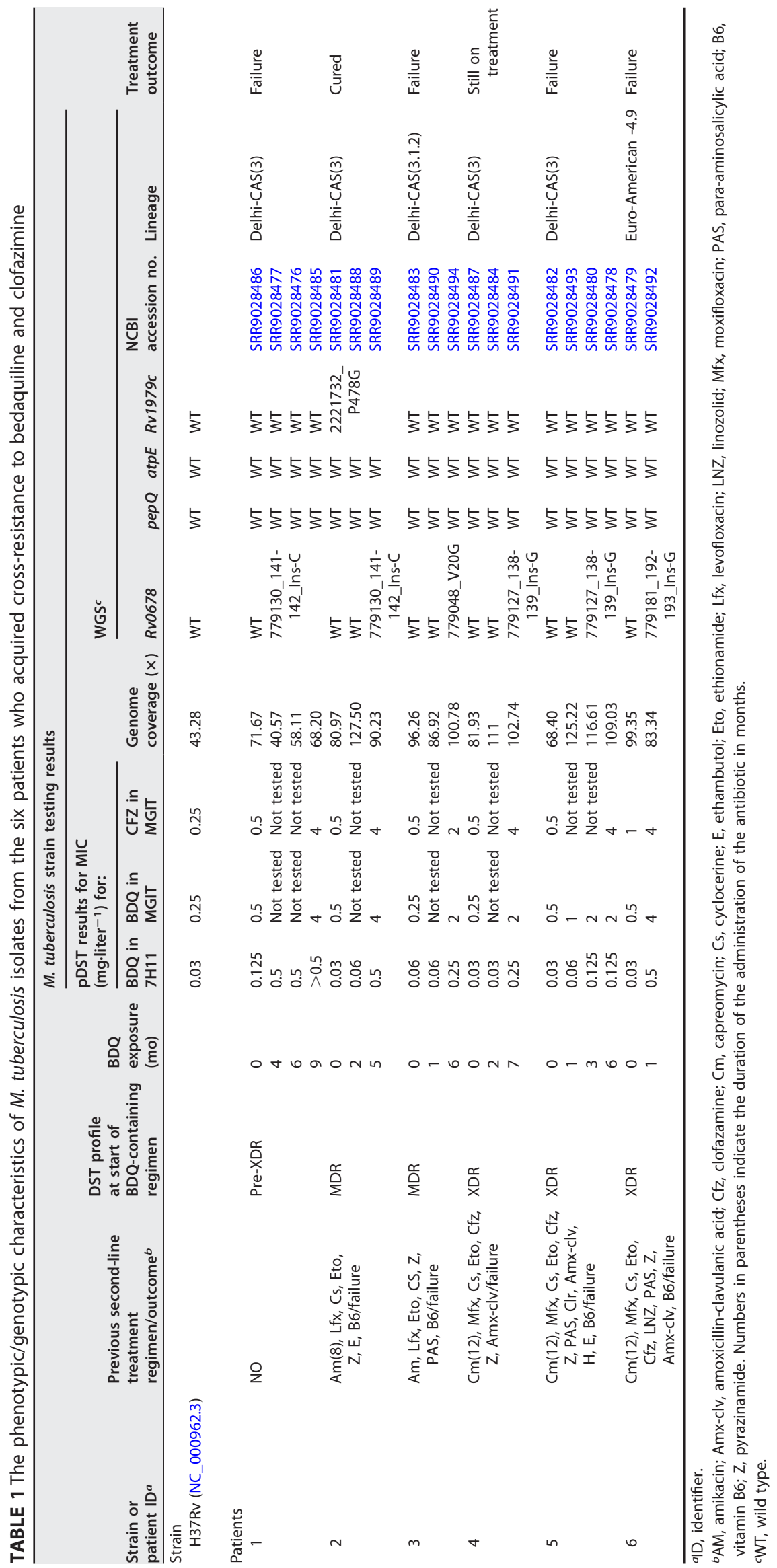


clofazimine-containing regimen. For both patients, no difference was seen in the bedaquiline MIC between the baseline isolate and the isolates collected after two and one month of bedaquiline-containing treatment. A subsequent isolate from patient 2 at the fifth month of treatment became resistant to both bedaquiline and clofazimine, and WGS analysis showed the appearance of an insertion at position 141 to 142 of Rv0678. Similarly, isolate from patient 3 after 6 months of bedaquiline showed 8- and 4-fold increases in bedaquiline and clofazimine MICs in mycobacterial growth indicator tubes (MGITs), and a V20G mutation in Rv0678 was detected. Moreover, the $M$. tuberculosis strains from patient 2 at baseline and at two and five months of treatment carried a P478G mutation in Rv1979c, suggesting that this mutation has no effect on clofazimine resistance.

Patients 4, 5, and 6 were XDR TB cases failing a second-line treatment regimen containing clofazimine administered for 20,14 , and 12 months, respectively. Baseline isolates of all three cases had a bedaquiline MIC of $0.03 \mathrm{mg}^{\circ}$ liter $^{-1}$ in $7 \mathrm{H} 11$ and showed no detectable mutations in Rv0678, pepQ, atpE, and Rv1979c. The follow-up isolates from patients 4 and 5 collected at months 6 and 5 during treatment showed an increase in bedaquiline and clofazimine MIC associated with an insertion at position 138 to 139 of Rv0678 (genomic position 779127). Patient 6 showed a substantial increase in MICs for both bedaquiline and clofazimine (8-fold for bedaquiline, 4-fold for clofazimine) after 1 month of therapy, and we detected an insertion in Rv0678 at a variant frequency of $12.7 \%$ that was not present at baseline. This patient ultimately failed treatment.

Breakpoints for bedaquiline are still provisional $(12,13)$, and patients are started on treatment without susceptibility tests. We adopted $0.25 \mathrm{mg} \cdot$ liter $^{-1}$ as the susceptibility breakpoint in $7 \mathrm{H} 10$ and $7 \mathrm{H} 11(14,15)$, which is above the MIC that inhibits $90 \%$ of the isolates or strains $\left(0.125 \mathrm{mg} \cdot\right.$ liter $\left.^{-1}\right)$. However, with this breakpoint, the strains collected from two patients reported as "failures" at the end of treatment (patient 4, MIC of $0.25 \mathrm{mg} \cdot$ liter $^{-1}$, 8-fold higher than baseline; and patient 5, MIC of $0.125 \mathrm{mg}^{\circ}$ liter $^{-1}$, 4-fold higher than baseline) would have been categorized as susceptible. This finding indicates that monitoring MICs during treatment could be a better predictor for failure than single testing at the critical concentration. The genetic basis of resistance to bedaquiline is still the subject of much uncertainty. WGS analyses in different studies showed that bedaquiline-clofazimine cross-resistance arises through mutations in Rv0678 $(8,9,10)$ and pepQ (11). In this study, we show that the increase in the MICs to bedaquiline and clofazimine could be explained by mutations in Rv0678 emerging during therapy. As reported in Table 1, we observed four different mutations, and three of these (138-139_Ins-G, 141-142_Ins-C, and 192-193_Ins-G) were previously reported as associated with bedaquiline resistance in $M$. tuberculosis clinical strains $(10,16)$. We show that the same mutations are associated with clofazimine resistance. As a result, regimens that contain both drugs might have to be reconsidered when these mutations are identified in order to reduce the risk of treatment failure for patients and the transmission of such strains in the community. Altogether, these data show that resistance to bedaquiline emerges during treatment and emphasize the importance of using MIC coupled, whenever possible, with WGS in national programs implementing bedaquiline for the treatment of MDR/XDR TB to monitor the emergence of resistance. Moreover, the collection of genomic data on mutations associated with bedaquiline and clofazimine resistance is crucial to lead future development of tools for fast detection of resistance. Introducing drugs without proper diagnostics to monitor drug resistance may lead to the amplification of hardly treatable cases.

Accession number(s). Raw sequencing reads have been deposited at BioProject at NCBI (project accession number PRJNA540911).

\section{ACKNOWLEDGMENTS}

We thank Andrea Maurizio Cabibbe for his guidance and Anna Dean and Matteo Zignol for their critical revision during the preparation of the manuscript.

We declare no conflict of interest. 
This study was funded by a WHO project to NRL/NTP Pakistan and SRL-Milano on the surveillance of acquisition of resistance to bedaquiline in Pakistan.

\section{REFERENCES}

1. WHO. 2019. WHO consolidated guidelines on drug-resistant tuberculosis treatment. World Health Organization, Geneva, Switerzland. License CC BY-NC-SA 3.0 IGO.

2. Schnippel K, Ndjeka N, Maartens G, Meintjes G, Master I, Ismail N, Hughes J, Ferreira H, Padanilam X, Romero R, Te Riele J, Conradie F. 2018. Effect of bedaquiline on mortality in South African patients with drug-resistant tuberculosis: a retrospective cohort study. Lancet Respir Med 6:699-706. https://doi.org/10.1016/S2213-2600(18)30235-2.

3. Borisov SE, Dheda K, Enwerem M, Romero Leyet R, D'Ambrosio L, Centis R, Sotgiu G, Tiberi S, Alffenaar J-W, Maryandyshev A, Belilovski E, Ganatra S, Skrahina A, Akkerman O, Aleksa A, Amale R, Artsukevich J, Bruchfeld J, Caminero JA, Carpena Martinez I, Codecasa L, Dalcolmo M, Denholm J, Douglas P, Duarte R, Esmail A, Fadul M, Filippov A, Davies Forsman L, Gaga M, Garcia-Fuertes J-A, García-García J-M, Gualano G, Jonsson J, Kunst H, Lau JS, Lazaro Mastrapa B, Teran Troya JL, Manga S, Manika K, González Montaner P, Mullerpattan J, Oelofse S, Ortelli M, Palmero DJ, Palmieri F, Papalia A, Papavasileiou A, Payen M-C, et al. 2017. Effectiveness and safety of bedaquiline-containing regimens in the treatment of MDR- and XDR-TB: a multicentre study. Eur Respir J 49:1700387. https:// doi.org/10.1183/13993003.00387-2017.

4. Honeyborne I, Lipman M, Zumla A, McHugh TD. 2019. The changing treatment landscape for MDR/XDR-TB - can current clinical trials revolutionise and inform a brave new world? Int J Infect Dis 80:S23-S28. https://doi.org/10.1016/j.ijid.2019.02.006.

5. Akkerman O, Aleksa A, Alffenaar J-W, Al-Marzouqi NH, Arias-Guillén M, Belilovski E, Bernal E, Boeree MJ, Borisov SE, Bruchfeld J, Cadiñanos Loidi J, Cai Q, Caminero JA, Cebrian Gallardo JJ, Centis R, Codecasa LR, D'Ambrosio L, Dalcolmo M, Danila E, Dara M, Davidavičienė E, Davies Forsman L, De Los Rios Jefe J, Denholm J, Duarte R, Elamin SE, Ferrarese M, Filippov A, Ganatra S, Garcia A, García-García J-M, Gayoso R, Giraldo Montoya AM, Gomez Rosso RG, Gualano G, Hoefsloot W, IlievskaPoposka B, Jonsson J, Khimova E, Kuksa L, Kunst H, Laniado-Laborín R, Li Y, Magis-Escurra C, Manfrin V, Manga S, Marchese V, Martínez Robles E, Maryandyshev A, Matteelli A, et al. 2019. Surveillance of adverse events in the treatment of drug-resistant tuberculosis: A global feasibility study. Int J Infect Dis 83:72-76. https://doi.org/10.1016/j.ijid.2019.03.036.

6. WHO. 2018. Technical manual for drug susceptibility testing of medicines used in the treatment of tuberculosis. World Health Organization, Geneva, Switzerland. License CC BY-NC-SA 3.0 IGO.

7. Kohl TA, Utpatel C, Schleusener V, De Filippo MR, Beckert P, Cirillo DM, Niemann S. 2018. MTBseq: a comprehensive pipeline for whole genome sequence analysis of Mycobacterium tuberculosis complex isolates. PeerJ 6:e5895. https://doi.org/10.7717/peerj.5895.

8. Hartkoorn RC, Uplekar S, Cole ST. 2014. Cross-resistance between clofazimine and bedaquiline through upregulation of MmpL5 in Mycobacterium tuberculosis. Antimicrob Agents Chemother 58:2979. https://doi .org/10.1128/AAC.00037-14.

9. Hoffmann H, Kohl TA, Hofmann-Thiel S, Merker M, Beckert P, Jaton $\mathrm{K}$, Nedialkova L, Sahalchyk E, Rothe T, Keller PM, Niemann S. 2016. Delamanid and bedaquiline resistance in Mycobacterium tuberculosis ancestral Beijing genotype causing extensively drug-resistant tuberculosis in a Tibetan refugee. Am J Respir Crit Care Med 193:337-340. https://doi.org/ 10.1164/rccm.201502-0372LE.

10. Ismail NA, Omar SV, Joseph L, Govender N, Blows L, Ismail F, Koornhof H, Dreyer AW, Kaniga K, Ndjeka N. 2018. Defining bedaquiline susceptibility, resistance, cross-resistance and associated genetic determinants: a retrospective cohort study. EBioMedicine 28:136-142. https://doi.org/10 .1016/j.ebiom.2018.01.005.

11. Almeida D, loerger T, Tyagi S, Li S-Y, Mdluli K, Andries K, Grosset J, Sacchettini J, Nuermberger E. 2016. Mutations in pepQ confer low-level resistance to bedaquiline and clofazimine in Mycobacterium tuberculosis. Antimicrob Agents Chemother 60:4590. https://doi.org/10.1128/AAC .00753-16.

12. Salfinger M, Migliori GB. 2015. Bedaquiline: 10 years later, the drug susceptibility testing protocol is still pending. Eur Respir J 45:317. https://doi.org/10.1183/09031936.00199814.

13. Köser CU, Maurer FP, Kranzer K. 2019. 'Those who cannot remember the past are condemned to repeat it': drug-susceptibility testing for bedaquiline and delamanid. Int J Infect Dis 80:S32-S35. https://doi.org/10 .1016/j.ijid.2019.02.027.

14. EUCAST. 1 January 2018. Breakpoint tables for interpretation of MICs and zone diameters. Version 8.0. http://www.eucast.org/fileadmin/src/media/ PDFs/EUCAST_files/Breakpoint_tables/v_8.0_Breakpoint_Tables.pdf.

15. WHO. 2018. Technical report on critical concentrations for TB drug susceptibility testing of medicines used in the treatment of drugresistant TB. World Health Organization, Geneva, Switzerland. License CC BY-NC-SA 3.0 IGO.

16. de Vos M, Ley SD, Wiggins KB, Derendinger B, Dippenaar A, Grobbelaar M, Reuter A, Dolby $T$, Burns S, Schito $M$, Engelthaler DM, Metcalfe J, Theron G, van Rie A, Posey J, Warren R, Cox H. 2019. Bedaquiline microheteroresistance after cessation of tuberculosis treatment. $\mathrm{N}$ Engl J Med 380:2178-2180. https://doi.org/10.1056/NEJMc1815121. 